\title{
ESTUDO CINÉTICO DA DECOMPOSIÇÃO TÉRMICA DO PENTAERETRITOL-TETRANITRADO (PETN)
}

\author{
Gilson da Silva* \\ Divisão de Química I, Diretoria de Patentes, Instituto Nacional da Propriedade Industrial, Rua Mayrink Veiga, 9 , \\ 20090-210 Rio de Janeiro - RJ, Brasil \\ Nanci Miyeko Nakamura \\ Divisão de Sistemas de Defesa, Instituto de Aeronáutica e Espaço, Comando-Geral de Tecnologia Aeroespacial, Praça Marechal \\ do Ar Eduardo Gomes, 50, 12228-904 São José dos Campos - SP, Brasil \\ Koshun Iha \\ Instituto Tecnológico de Aeronáutica, Comando-Geral de Tecnologia Aeroespacial, Pr. Marechal do Ar Eduardo Gomes, 50, \\ 12228-904 São José dos Campos - SP, Brasil
}

Recebido em 25/2/08; aceito em 12/6/08; publicado na web em 10/11/08

\begin{abstract}
KINETIC STUDY OF THE THERMAL DECOMPOSITION OF PENTAERYTHRITOL-TETRANITRATE (PETN). The pentaerythritol-tetranitrate (PETN) is a nitroether used in explosives and propellant formulations. Due to its suitable properties, PETN is used in booster manufacture. Knowing the thermal decomposition behavior of an energetic material is very important for storage and manipulation, and the purpose of this work is to study the kinetic parameters of the decomposition of PETN, compare the results with literature data and to study the decomposition activation energy differences between two crystalline forms of PETN (tetragonal and needle) by means of differential scanning calorimetry (DSC). Fourier transform infrared spectroscopy (FT-IR) is used to study the two crystalline forms.
\end{abstract}

Keywords: PETN; DSC; FT-IR.

\section{INTRODUÇÃO}

As diversas técnicas termoanalíticas utilizadas isoladamente ou em conjunto com outras técnicas, tais como espectroscopia no infravermelho (IR), espectroscopia de massas (MS), cromatografia a gás (CG) ou cromatografia líquida de alta eficiência (CLAE), apresentamse como ferramentas importantes no estudo dos materiais energéticos. ${ }^{1}$ A análise térmica diferencial (DTA), a calorimetria exploratória diferencial (DSC) e a termogravimetria (TG) podem ser utilizadas, por exemplo, na comparação dos mecanismos físico-químicos relativos a processos de decomposição térmica, transição de fase cristalina ${ }^{2}$ ou no estudo e desenvolvimento de novos compostos.

Técnicas termoanalíticas têm sido ainda amplamente usadas na caracterização e quantificação de explosivos. ${ }^{3,4}$ DSC, técnica utilizada neste trabalho, permite, entre outras informações, determinar temperaturas de fusão, de decomposição, além de fornecer dados sobre a estabilidade térmica de compostos. Tem como vantagens o uso de quantidade reduzida de amostra e rapidez, além de permitir a obtenção das informações necessárias para determinação dos parâmetros cinéticos de uma reação. . $^{2,5,6}$

Um importante ponto de partida para o desenvolvimento seguro de materiais energéticos e métodos que especifiquem suas propriedades de risco consiste no estudo da relação entre a estrutura molecular desses materiais e a sensibilidade elétrica, ao impacto, atrito, e calor. ${ }^{7}$ Portanto, o comportamento térmico de materiais energéticos é muito importante para garantia de sua produção, armazenagem e manuseio, ${ }^{5}$ além de permitir o entendimento do mecanismo de explosão. ${ }^{8}$

Dentre os nitratos cristalinos mais utilizados em formulações de propelentes e explosivos encontram-se os nitratos de amônio, potássio, sódio, triaminoguanidina (TAGN) e o pentaeretritol-tetranitrado (PETN). ${ }^{9}$

\footnotetext{
*e-mail: gilsondasilva@uol.com.br
}

O PETN é um éter nitrado ${ }^{10}$ que possui diversos nomes tradicionais em vários países: PETN, Pentrite, Penta, Pentrit, Nitropenta, NP Pentryt, Tem. ${ }^{11,12}$ É uma substância cristalina branca que possui temperatura de fusão entre 140 e $141{ }^{\circ} \mathrm{C} .{ }^{10,11}$ Insolúvel em água, o PETN apresenta solubilidade em uma grande variedade de solventes orgânicos e, particularmente, em acetona. ${ }^{12}$ A Figura 1S (material suplementar) ilustra a estrutura molecular do PETN.

Os principais produtos gasosos da decomposição do PETN, segundo Urbanski, ${ }^{11}$ são $\mathrm{NO}_{2}, \mathrm{NO}, \mathrm{N}_{2} \mathrm{O}, \mathrm{N}_{2}, \mathrm{CO}_{2}, \mathrm{CO}, \mathrm{He} \mathrm{O}_{2}$. Os teores desses gases variam em função do tipo de estímulo ao qual o PETN é submetido (impacto, detonação ou decomposição térmica).

Este composto não é muito sensível à fricção, mas é caracterizado por alta sensibilidade à iniciação por explosão, detonado por $0,01 \mathrm{~g}$ de azoteto de chumbo. ${ }^{11}$ Tal característica faz dele um excelente explosivo para a transmissão de detonação de um explosivo de iniciação para um alto explosivo (explosivo secundário).

Esse mecanismo de transmissão de detonação é muito usado em sistemas de "trem explosivo", ${ }^{3}$ no qual diversos explosivos são colocados em contato, de forma que o mais sensível (explosivo primário - iniciado por meio térmico, elétrico, etc.) inicie um segundo explosivo (reforçador) que, por sua vez, transmite a detonação a um terceiro explosivo de grande potência (explosivo secundário). 3.13

Anomalias associadas ao calor de fusão do PETN cristalizado por diferentes métodos foram estudadas por diversas técnicas, ${ }^{14}$ sendo que a microscopia revelou a existência de, no mínimo, três tipos cristalinos: tetragonal, agulhada e placas irregulares; onde os calores de fusão são, respectivamente, 152,8-156,6; 152,8-157,8 e $132,7-139,0 \mathrm{~J} / \mathrm{g}^{14}$

Cristais superfinos de PETN, em forma agulhada, são obtidos por sua dissolução em acetona a quente e precipitados por adição de água fria, ${ }^{12}$ sendo utilizados em detonadores.

Existem diversos métodos para determinação dos parâmetros cinéticos de uma reação, aos quais se aplicam os dados obtidos com 
o auxílio de um calorímetro exploratório diferencial (DSC) ou, em alguns casos, analisador térmico diferencial (DTA). Entre estes, são citados os de Kissinger, Freeman e Carroll, Friedman, Coats e Redfren, Ozawa, e outros. Entretanto, a ASTM E 698 recomenda a utilização dos métodos de Kissinger e Ozawa na determinação de parâmetros cinéticos. ${ }^{15}$

Segundo Kissinger, o cálculo dos parâmetros cinéticos é efetuado por meio da Equação 1:6

$\ln \left(\frac{\phi}{T_{p}^{2}}\right)=\ln \left(\frac{A R}{T_{p}}\right)-\frac{E_{a}}{R T_{p}}$

em que: $\phi$ é a razão de aquecimento $\left(\mathrm{K} \mathrm{s}^{-1}\right) ; \mathrm{T}_{\mathrm{p}}$, a temperatura absoluta do pico em cada razão de aquecimento $(\mathrm{K}) ; \mathrm{A}$, o fator pré-exponencial de Arrhenius $\left(\mathrm{s}^{-1}\right) ; \mathrm{R}$, a constante dos gases $\left(8,3145 \mathrm{~J} \mathrm{~mol}^{-1} \mathrm{~K}^{-1}\right)$ e $\mathrm{E}_{\mathrm{a}}$, a energia de ativação da reação $\left(\mathrm{J} \mathrm{mol}^{-1}\right)$.

A energia de ativação pode ser obtida por meio de dados de ensaios DSC em diferentes razões de aquecimento, onde ocorrem deslocamentos na temperatura do pico da reação.

A Equação de Kissinger pode ser associada à equação da reta (y $=\mathrm{mx}+\mathrm{b})$, ou seja, $\mathrm{y}=\ln \left(\phi / \mathrm{T}_{\mathrm{p}}{ }^{2}\right), \mathrm{m}=-\mathrm{E}_{\mathrm{a}} / \mathrm{R}, \mathrm{x}=1 / \mathrm{T}_{\mathrm{p}}$ e $\mathrm{b}=\ln (\mathrm{AR} /$ $\mathrm{T}_{\mathrm{p}}$ ). Com isso, o valor de $\mathrm{E}_{\mathrm{a}}$ pode ser determinado por meio de um gráfico de $\ln \left(\phi / \mathrm{T}_{\mathrm{p}}{ }^{2}\right)$ versus $1 / \mathrm{T}_{\mathrm{p}}$, resultando numa reta cujo coeficiente angular é $-\mathrm{E}_{\mathrm{a}} / \mathrm{R}$, e o coeficiente linear é ln $\left(\mathrm{AR} / \mathrm{T}_{\mathrm{p}}\right)$.

O método de Ozawa é outra forma de obter os parâmetros cinéticos da reação. ${ }^{5,16,17}$ Neste método, o logaritmo da razão de aquecimento $(\phi)$ é colocado em função do inverso da temperatura máxima do pico de transição. A energia de ativação também é calculada por esse método, por meio do coeficiente angular da reta média entre os pontos, e a extrapolação dessa reta fornece o fator pré-exponencial (A). Segundo Ozawa, utiliza-se para o cálculo dos parâmetros de Arrhenius a Equação 2:

$E_{a}=-2,19 R\left[\frac{d \log _{10} \phi}{1 / T_{p}}\right]$

No entanto, a utilização do método de Ozawa requer um refinamento para a determinação da $\mathrm{E}_{\mathrm{a}}$, que é realizado por meio da aproximação de Doyle. ${ }^{15} \mathrm{O}$ quociente $\mathrm{E}_{\mathrm{a}} / \mathrm{RT}_{\mathrm{p}}$ é calculado, onde $\mathrm{T}_{\mathrm{p}}$ é a temperatura máxima do pico obtido para $\phi$ central da faixa das razões de aquecimento adotadas, e o valor do fator D é então encontrado por consulta a uma tabela disponível na ASTM E $698 .{ }^{15}$ O novo valor de E é obtido por meio da Equação 3:15

$E_{a}=\left(-2,303 \frac{R}{D}\right)\left[\frac{d \log _{10} \phi}{1 / T_{p}}\right]$

A literatura recomenda ainda que a determinação do fator préexponencial de Arrhenius seja calculada por meio da Equação 4:15

$A=\frac{\phi E_{a} e^{\frac{E a}{R T}}}{R T_{p}{ }^{2}}$

onde $\phi$ também é a razão de aquecimento central da faixa escolhida para estudo.

O objetivo deste trabalho foi determinar os parâmetros de Arrhenius (energia de ativação e fator pré-exponencial) para a reação de decomposição do PETN nas formas cristalinas tetragonal e agulhada, comparando os resultados entre si e com os encontrados na literatura.

\section{PARTE EXPERIMENTAL}

O estudo foi realizado com PETN em duas formas cristalinas diferentes, tetragonal e agulhada. O produto na forma tetragonal foi adquirido comercialmente da IMBEL, enquanto que a forma agulhada foi obtida por meio da recristalização do PETN tetragonal em sistema acetona/água.

Com base no fato de que o PETN apresenta fusão em aproximadamente $140{ }^{\circ} \mathrm{C}$, ao se decompor o material está em estado líquido. ${ }^{12}$ Portanto, considerou-se que a distribuição granulométrica do mesmo não influencia o mecanismo de decomposição, uma vez que a decomposição do material ocorre em estado líquido.

A caracterização espectrométrica foi realizada em um espectrômetro FT-IR Spectrum 2000 Perkin-Elmer, região de 4000 a $400 \mathrm{~cm}^{-1}$, resolução $4 \mathrm{~cm}^{-1}$, ganho 1 e 40 varreduras. As amostras foram analisadas segundo a técnica de transmissão, pastilha de $\mathrm{KBr}$ na proporção 0,8:400 mg. Os espectros da amostra e material de referência foram comparados entre si e com bandas analíticas de um espectro FT-IR de referência do material. Foi utilizada ainda microscopia FT-IR para obtenção de micrografias dos cristais de PETN.

As curvas DSC foram obtidas em um calorímetro DSC-7 PerkinElmer. O equipamento foi calibrado em cada razão de aquecimento com zinco e índio. As massas das amostras foram aproximadamente de $1,5 \mathrm{mg}$. Os intervalos de aquecimento ficaram entre 50 e $250^{\circ} \mathrm{C}$, sendo as amostras submetidas a razões de aquecimento $(\phi)$ de 10,12, 15, 17 e $20^{\circ} \mathrm{C} \mathrm{min}{ }^{-1}$. As curvas foram obtidas sob fluxo de nitrogênio gasoso (40 mL/min) e à pressão atmosférica, ou seja, as tampas das cápsulas DSC foram perfuradas de modo a não confinar o explosivo.

\section{RESULTADOS E DISCUSSÃO}

A espectroscopia na região do infravermelho foi utilizada para verificar possíveis alterações espectrais em função da forma cristalina, tal como observado em outros explosivos como, por exemplo, o octogênio (HMX). ${ }^{18}$ Os espectros do PETN nas duas formas cristalinas foram comparados entre si e com um espectro de referência do material puro existente nos arquivos do Laboratório Instrumental da Divisão de Química (AQI/IAE).

As principais bandas de absorção observadas foram: $1658 \mathrm{~cm}^{-1}$ $\left(v_{\mathrm{a}} \mathrm{NO}_{2}\right), 1285 \mathrm{~cm}^{-1}\left(\mathrm{v}_{\mathrm{s}} \mathrm{NO}_{2}\right), 1272 \mathrm{~cm}^{-1}\left(\mathrm{v}_{\mathrm{s}} \mathrm{NO}_{2}\right), 869$ e $852 \mathrm{~cm}^{-1}$ ( $\vee \mathrm{NO}), 754$ e $703 \mathrm{~cm}^{-1}\left(\delta_{\mathrm{a}}\right.$ e $\left.\delta_{\mathrm{s}} \mathrm{NO}\right)$. Nos espectros, apresentados na Figura 1, não foram observados deslocamentos ou ausências de bandas de absorção do PETN em função de sua estrutura cristalina. Dessa forma, a diferenciação entre a estrutura cristalina tetragonal e a agulhada do PETN não é possível por FT-IR, utilizando técnica de transmissão em pastilha de $\mathrm{KBr}$.

Por meio da microscopia FT-IR foi possível a ampliação da imagem dos cristais em aproximadamente 600 vezes, o que possibilitou verificar que o PETN tetragonal (Figura $2 \mathrm{Sa}$ ) apresenta estrutura poliédrica com tamanhos mais uniformes, enquanto que o PETN agulhado (Figura $2 \mathrm{Sb}$ ) possui cristais alongados e de tamanhos irregulares. A Figura 2S (material suplementar) apresenta as fotomicrografias do PETN nas duas formas cristalinas estudadas.

De acordo com o comportamento esperado para ambas as amostras, os ensaios DSC mostraram que a temperatura máxima de decomposição $\left(\mathrm{T}_{\mathrm{p}}\right)$ do material variou em função do aumento da razão de aquecimento $(\phi)$ adotada no ensaio, aumentando conforme a razão de aquecimento foi aumentada. A Figura 2 apresenta o perfil das curvas DSC referentes à decomposição do PETN na forma tetragonal, enquanto a Tabela 1 apresenta os dados obtidos por meio desses ensaios.

O perfil das curvas térmicas do PETN agulhado foi muito similar ao do tetragonal, uma vez que na temperatura de decomposição o 


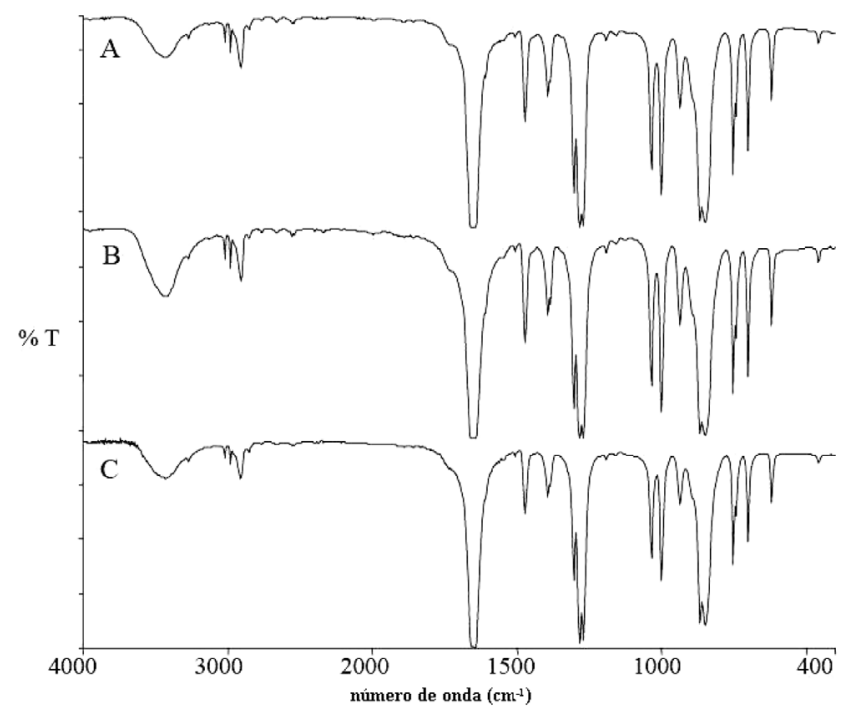

Figura 1. Espectro FT-IR de transmissão em pastilha de KBr do PETN: A) PETN sob a forma cristalina agulhada, B) PETN sob a forma cristalina tetragonal e C) PETN existente nos arquivos do Laboratório Instrumental

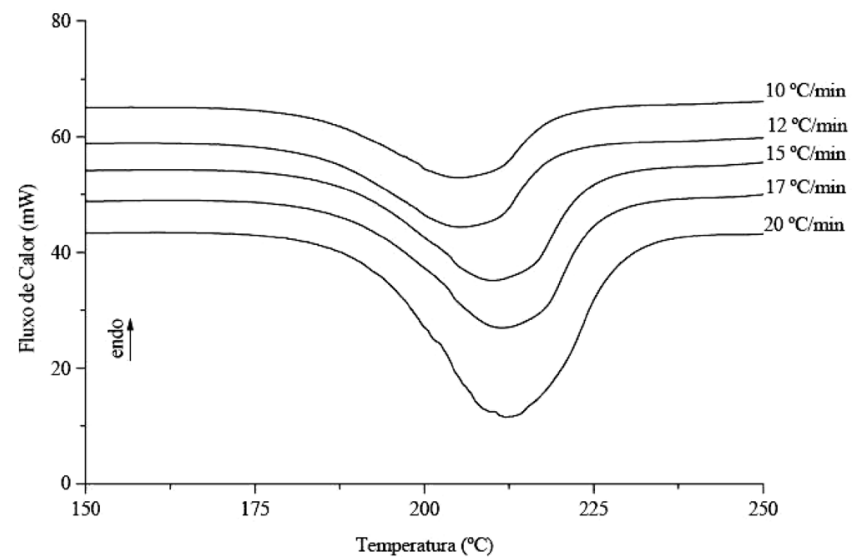

Figura 2. Curvas DSC da decomposição térmica do PETN sob a forma cristalina tetragonal em atmosfera dinâmica de $\mathrm{N}_{2}$

Tabela 1. Dados obtidos com as curvas DSC da decomposição térmica do PETN sob a forma tetragonal

\begin{tabular}{lcc}
\hline$\phi\left({ }^{\circ} \mathrm{C} / \mathrm{min}\right)$ & $\begin{array}{c}\text { Temperatura do pico de } \\
\text { decomposição }-\mathrm{T}_{\mathrm{p}}(\mathrm{K})\end{array}$ & $\begin{array}{c}\text { Entalpia de decomposição } \\
(\mathrm{J} / \mathrm{g})\end{array}$ \\
\hline 10 & $478,4 \pm 0,1$ & $-930 \pm 26$ \\
12 & $480,3 \pm 0,1$ & $-941 \pm 5$ \\
15 & $484,5 \pm 0,1$ & $-929 \pm 11$ \\
17 & $484,6 \pm 0,2$ & $-928 \pm 7$ \\
20 & $486,7 \pm 0,1$ & $-912 \pm 25$ \\
\hline
\end{tabular}

PETN se encontra completamente fundido. As curvas DSC do PETN agulhado são apresentadas na Figura 3 e os dados obtidos nesses ensaios, na Tabela 2.

\section{Cálculo dos parâmetros de Arrhenius pelo método de Kissinger $^{15}$}

Os dados referentes às curvas DSC apresentados nas Tabelas $1 \mathrm{e}$ 2 foram submetidos ao método de Kissinger, Equação 1. As Figuras

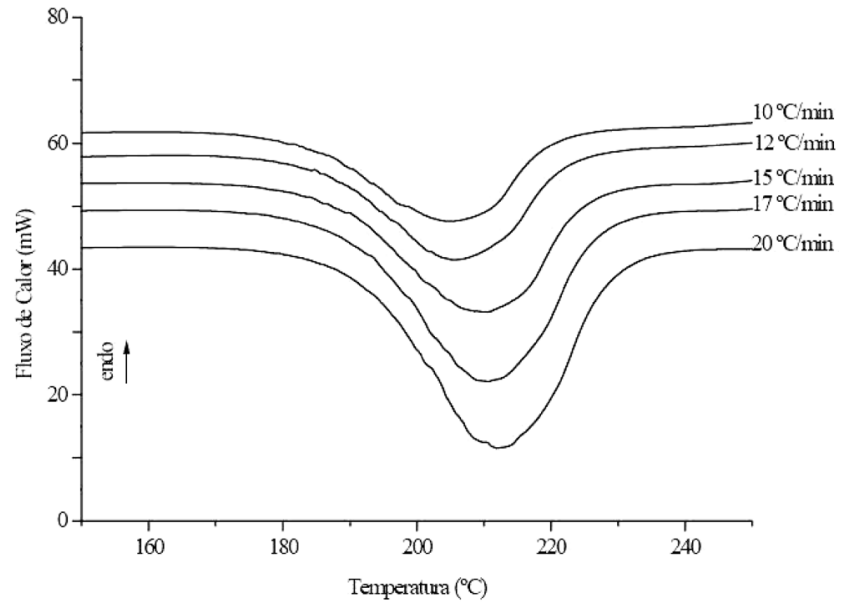

Figura 3. Curvas DSC da decomposição térmica do PETN sob a forma cristalina agulhada em atmosfera dinâmica de $\mathrm{N}_{2}$

Tabela 2. Dados obtidos com as curvas DSC da decomposição térmica do PETN sob a forma agulhada

\begin{tabular}{lcc}
\hline$\phi\left({ }^{\circ} \mathrm{C} / \mathrm{min}\right)$ & $\begin{array}{c}\text { Temperatura do pico de } \\
\text { decomposição }-\mathrm{T}_{\mathrm{p}}(\mathrm{K})\end{array}$ & $\begin{array}{c}\text { Entalpia de decomposição } \\
\left(\mathrm{J} \mathrm{g}^{-1}\right)\end{array}$ \\
\hline 10 & $477,6 \pm 0,4$ & $-1048 \pm 74$ \\
12 & $479,3 \pm 0,3$ & $-1075 \pm 14$ \\
15 & $482,6 \pm 0,5$ & $-1047 \pm 18$ \\
17 & $483,7 \pm 0,1$ & $-1073 \pm 31$ \\
20 & $485,4 \pm 0,1$ & $-1084 \pm 48$ \\
\hline
\end{tabular}

4 e 5 apresentam os gráficos utilizados para determinação dos parâmetros de Arrhenius da decomposição térmica do PETN tetragonal e agulhado, respectivamente.

\section{Cálculo dos parâmetros de Arrhenius pelo método de Ozawa ${ }^{15}$}

Os dados referentes às curvas DSC apresentados nas Tabelas 1 e 2 foram submetidos ao método de Ozawa, Equação 2. As Figuras 6 e 7 apresentam os gráficos utilizados para determinação dos parâmetros de Arrhenius da decomposição térmica do PETN tetragonal e agulhado, respectivamente.

A Tabela 3 apresenta os valores dos coeficientes angulares obtidos por regressão linear dos pontos (Figuras 4-7), o coeficiente de determinação $\left(\mathrm{R}^{2}\right)$ para cada regressão linear, bem como os valores das

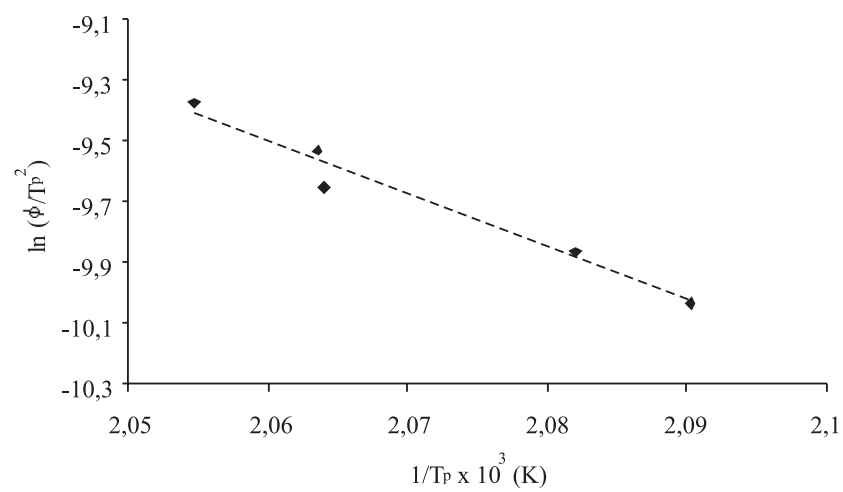

Figura 4. Gráfico referente ao método de Kissinger para determinação da energia de ativação da decomposição térmica do PETN sob a forma cristalina tetragonal 


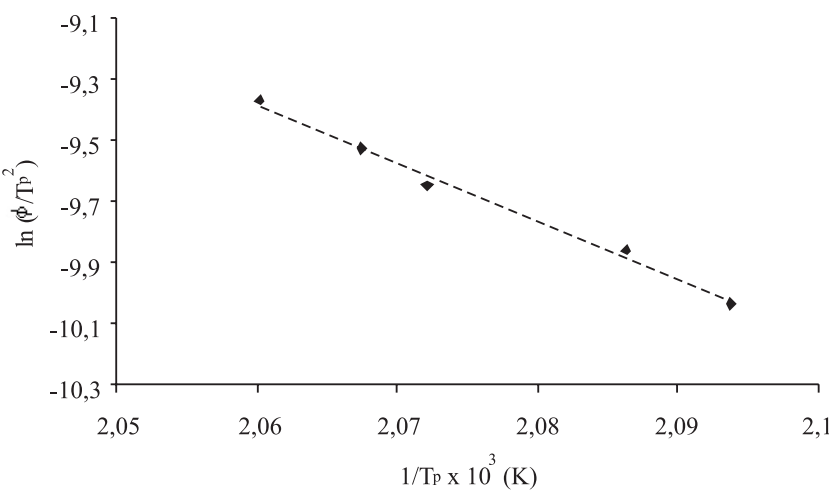

Figura 5. Gráfico referente ao método de Kissinger para determinação da energia de ativação da decomposição térmica do PETN sob a forma cristalina agulhada

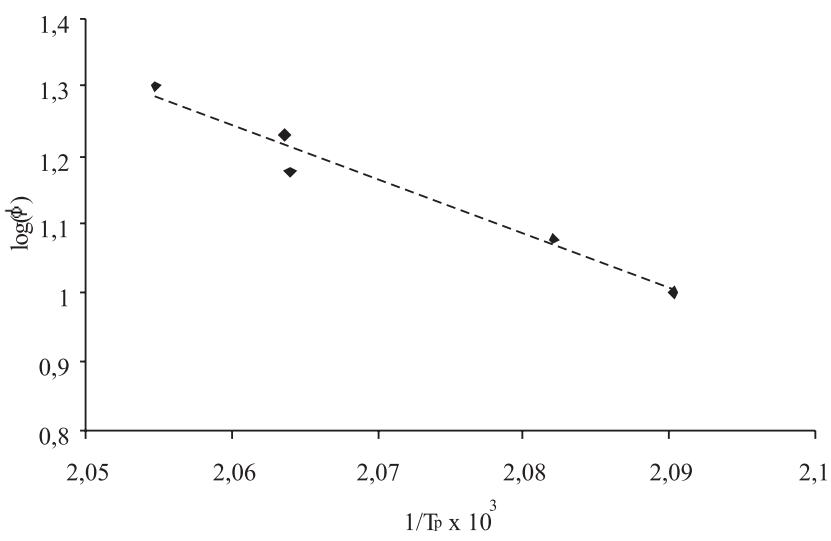

Figura 6. Gráfico referente ao método de Ozawa para determinação da energia de ativação da decomposição térmica do PETN sob a forma cristalina tetragonal

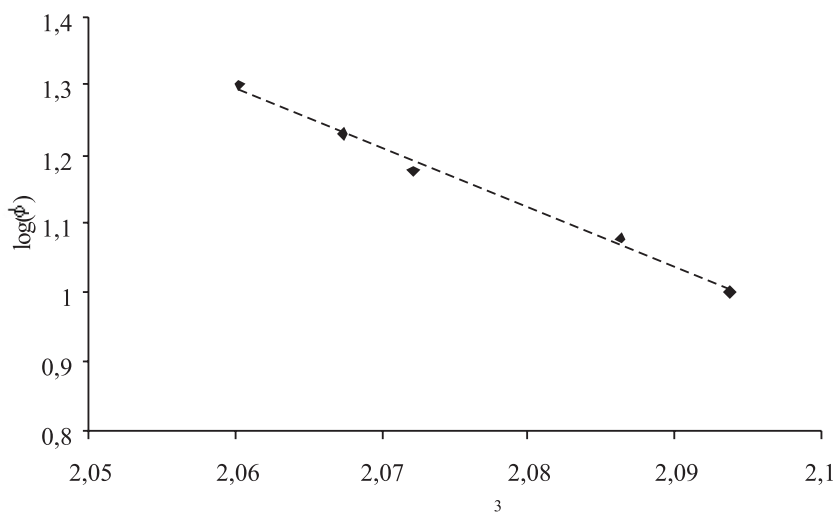

Figura 7. Gráfico referente ao método de Ozawa para determinação da energia de ativação da decomposição térmica do PETN sob a forma cristalina agulhada energias de ativação, referentes a ambos os métodos de determinação utilizados, e os fatores pré-exponenciais calculados.

A literatura revela diversos valores para a decomposição térmica do PETN, ${ }^{6,14,19,20}$ esses valores variam entre 116 e $196 \mathrm{~kJ} \mathrm{~mol}^{-1}$, e relata o uso de técnicas termoanalíticas para aquisição de dados necessários aos cálculos de energia de ativação. Portanto, os valores determinados por meio de ambos os métodos de cálculo cinético estão em concordância com os valores relatados na literatura. ${ }^{6,14,19,20}$

Com base no fato do PETN se decompor a partir do estado líquido, tendo em vista que sua fusão ocorre em aproximadamente $140{ }^{\circ} \mathrm{C}$, os valores das energias de ativação referentes ao processo de decomposição térmica deveriam ser mais próximos, bem como os valores das entalpias de decomposição, ver Tabelas 1 e 2. Entretanto, as diferenças observadas no comportamento térmico do PETN nas duas formas cristalinas estudadas podem ter sido geradas por pequenas variações de acidez oclusa nos cristais, ${ }^{21}$ alteradas graças ao processo de recristalização, a que foi submetido o PETN tetragonal para produção do PETN na forma agulhada, e/ou ainda a pequenos teores de impurezas que podem ter sido inseridos ou removidos por meio desse mesmo processo de recristalização.

\section{CONCLUSÕES}

As formas cristalinas tetragonal e agulhada do PETN, diferentemente de outros materiais energéticos, por exemplo HMX, não apresentam diferenças espectrométricas quando analisadas por FT-IR utilizando técnica de transmissão em pastilha de $\mathrm{KBr}$.

A energia de ativação determinada para o PETN por meio de ambos os métodos de cálculo de parâmetros cinéticos (Kissinger e Ozawa) está em concordância com a literatura, ${ }^{6,14,19,20}$ sendo os valores iguais, nos dois métodos de cálculo, para o material quando na mesma forma cristalina.

A diferença entre a energia de ativação do material na forma tetragonal e agulhada pode estar associada à acidez oclusa nos cristais e/ou a pequenos teores de impurezas provenientes do processo de recristalização do material. Tais condições poderiam alterar o mecanismo de decomposição térmica do PETN e, com isso, causar alterações nos valores da energia de ativação determinada.

\section{MATERIAL SUPLEMENTAR}

No material suplementar disponível em http://quimicanova. sbq.org.br, na forma de arquivo PDF, com acesso livre e gratuito, encontram-se as Figuras: 1S - Estrutura molecular do PETN; 2S Fotomicrografias obtidas por meio de microscópio FT-IR dos cristais de PETN sob as formas cristalinas tetragonal (A) e agulhada (B).

\section{AGRADECIMENTOS}

Ao quadro técnico do Laboratório Químico da Divisão de Sistemas de Defesa (ASD) e do Laboratório Instrumental da Divisão de Química (AQI) do Instituto de Aeronáutica e Espaço do ComandoGeral de Tecnologia Aeroespacial (CTA).

Tabela 3. Parâmetros cinéticos da decomposição térmica do PETN sob as formas cristalinas tetragonal e agulhada

\begin{tabular}{cccccccc}
\hline & \multicolumn{3}{c}{ Método de Kissinger } & \multicolumn{3}{c}{ Método de Ozawa $\left(\mathrm{s}^{-1}\right)$} \\
& $\mathrm{m}^{*}$ & $\mathrm{R}^{2}$ & $\mathrm{E}_{\mathrm{a}}\left(\mathrm{kJ} \mathrm{mol}^{-1}\right)$ & $\mathrm{m}^{*}$ & $\mathrm{R}^{2}$ & $\mathrm{E}_{\mathrm{a}}\left(\mathrm{kJmol}^{-1}\right)$ \\
\hline PETN $_{\mathrm{t}}$ & -17444 & 0,964 & 145 & -7995 & 0,968 & 145 & $4,8.10^{15}$ \\
PETN $_{\mathrm{a}}$ & -18881 & 0,992 & 157 & -8618 & 0,993 & 157 & $1,2.10^{17}$ \\
\hline
\end{tabular}

* Coeficiente angular 


\section{REFERÊNCIAS}

1. Andrade, J.; Iha, K.; Rocco, J. A. F. F.; Bezerra, E. M.; Iha, M. E. V. S.; Pinheiro, G. F. M. P.; Quim. Nova 2007, 30, 952.

2. Silva, G.; Mattos, E. C.; Nakamura, N. M.; Iha, K.; Quim. Nova 2004, 27, 889.

3. Silva, G.; Tese de Doutorado, Instituto Tecnológico de Aeronáutica, Brasil, 2007.

4. Silva, G.; Pinheiro, G. F. M.; Iha, K.; Dutra, R. C. L.; Takahashi, M. F. K.; Reis, T. B.; Quim. Nova 2006, 29, 681.

5. Silva, G.; Mattos, E. C.; Nakamura, N. M.; Iha, K.; Anais Assoc. Bras. Quim. 2003, 52, 81.

6. Lee, J. S.; Hsu, C. K.; Chang, C. L.; Thermochim. Acta 2002, 392-393, 173.

7. Chovancová, M.; Zeman, S.; Thermochim. Acta 2007, 460, 67.

8. Singh, G.; Kapoor, I. P. S.; Mannan, S. M.; Tiwari, S. K.; J. Hazard. Mater. 1999, A68, 155.

9. Kubota, N.; Propellants and Explosives, Wiley-VCH: Germany, 2002.

10. Filin, V. P.; Delkov, P. Y.; Garmasheva, N. V.; Timofeev, V. T.; Chemagina, I. V.; Shakhtorin, Y. A.; Taibinov, N. P.; Bagavetdinov, N. G.; Loboiko, B. G.; $37^{\text {th }}$ International Annual Conference of ICT, Karlsruhe, Germany, 2006.
11. Urbansk, T.; Chemistry and Technology of Explosives, Oxford: Pergamon, 1965, vol. 2 .

12. Silva, G.; Dissertação de Mestrado, Instituto Tecnológico de Aeronáutica, Brasil, 2004.

13. Quinlin, W. T.; Thorpe, R.; Lightfoot, J. M.; U.S. 2006/0025603, 2006.

14. Urbansk, T.; Chemistry and Technology of Explosives, Pergamon Press: Great Britain, 1984, vol. 4.

15. American Society For Testing And Materials; ASTM E 698-79: Arrhenius kinetic constants for thermally unstable material, Philadelphia, 1984.

16. Weese, R. K.; Maienschein, J. L.; Perrino, C. T.; Thermochim. Acta 2003, 401,1 .

17. Ozawa, T.; J. Therm. Anal. Calorim. 1970, 2, 301.

18. Mattos, E. C.; Tese de Mestrado, Instituto Tecnológico de Aeronáutica, Brasil, 2001.

19. Huang, C. C.; Wu, T. S.; Thermochim. Acta 1992, 204, 239.

20. Almada, S.; Campos, J.; Gois, J. C.; $11^{\text {th }}$ Symposium on Chemical Problems Connected with Stability of Explosives, Estocolmo, Suécia, 1998.

21. Somoza, C.; Estabrook, L. C.; U.S. 5.020.731 1991. 


\section{ESTUDO CINÉTICO DA DECOMPOSIÇÃO TÉRMICA DO PENTAERETRITOL-TETRANITRADO (PETN)}

\section{Gilson da Silva*}

Divisão de Química I, Diretoria de Patentes, Instituto Nacional da Propriedade Industrial, Rua Mayrink Veiga, 9 , 20090-210 Rio de Janeiro - RJ, Brasil

\section{Nanci Miyeko Nakamura}

Divisão de Sistemas de Defesa, Instituto de Aeronáutica e Espaço, Comando-Geral de Tecnologia Aeroespacial, Praça Marechal do Ar Eduardo Gomes, 50, 12228-904 São José dos Campos - SP, Brasil

\section{Koshun Iha}

Instituto Tecnológico de Aeronáutica, Comando-Geral de Tecnologia Aeroespacial, Pr. Marechal do Ar Eduardo Gomes, 50, 12228-904 São José dos Campos - SP, Brasil

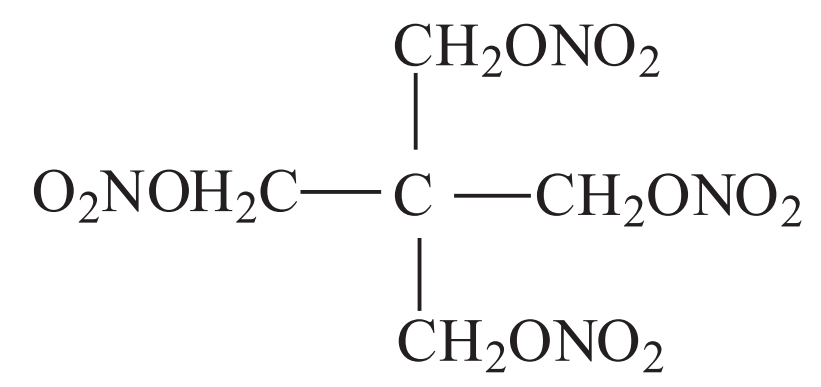

Figura 1S. Estrutura molecular do pentaeretritol-tetranitrado (PETN)
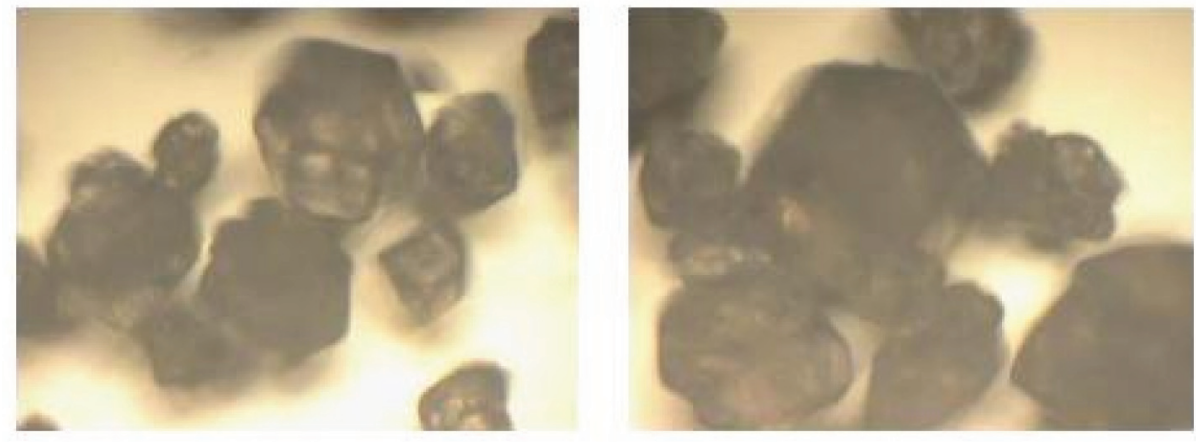

Cristais de PETN Tetragonal ( $A$ )
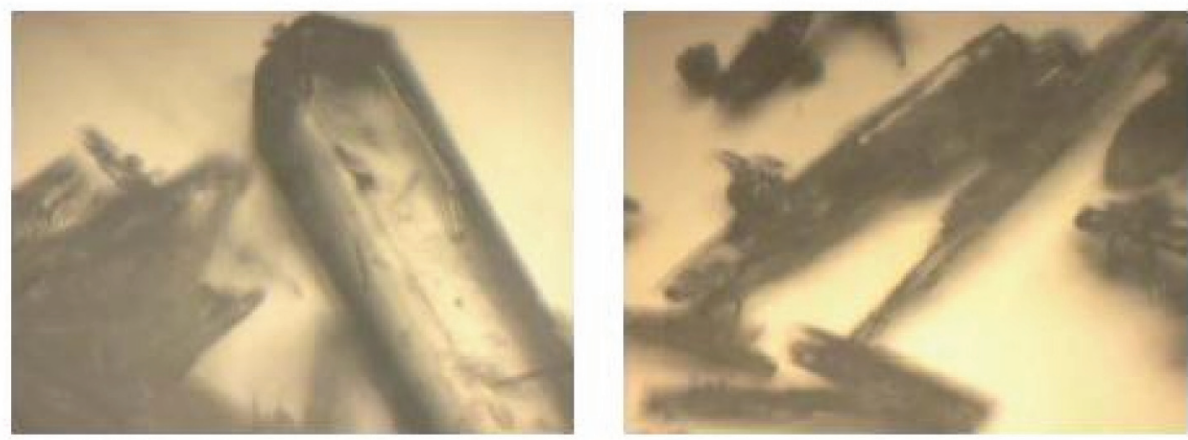

Cristais de PETN Agulhada (B)

Figura 2S. Fotomicrografias obtidas por meio de microscópio FT-IR dos cristais de PETN sob as formas cristalinas tetragonal (A) e agulhada (B) 